\title{
Mundmotorische Übungen in der Kindersprachtherapie
}

Eltern, deren Kinder sich wegen einer Aussprachestörung und/oder einer myofunktionellen Störung in sprachtherapeutischer/logopädischer Behandlung befinden, erleben häufig, dass während der Therapieeinheiten oder auch als Hausaufgaben mundund zungenmotorische Übungen durchgeführt werden (sollen). Eine generelle Durchführung dieser Übungen erscheint allerdings nicht sinnvoll, daher soll im Folgenden beschrieben werden, wann welche Übungen sinnvoll einzusetzen sind.

Zunächst erscheint es daher notwendig, die verschiedenen Formen der Aussprachestörungen und myofunktioneller Störungen zu erläutern: Unter einer myofunktionellen Störung versteht man eine Zungen-Fehlfunktion, die zu einem pathologischen, d.h. nicht regelrechten Schluckmuster des Kindes führt. Diese Zungen-Fehlfunktion ist auf ein muskuläres Ungleichgewicht oder eine muskuläre Schwäche im Bereich der Lippenund/oder Zungenmuskulatur zurückzuführen. Wurde eine myofunktionelle Störung diagnostiziert, ist der gezielte Einsatz von Zungen- und oder Lippenübungen ein sinnvolles Vorgehen, um ein muskuläres Gleichgewicht oder eine muskuläre Stärkung zu erreichen, das als Grundlage für die Überwindung der Zungen-Fehlfunktion dient. In der Regel findet ein therapeutisches Vorgehen nach Kittel [1] Anwendung, indem eine strukturierte Hierarchie von Muskelübungen vorgegeben ist.

Eine Aussprachestörung hingegen liegt dann vor, wenn ein Kind Laute auf nicht altersgerechte Weise verwendet, diese also fehlbildet, auslässt, vertauscht oder ersetzt, sodass die Verständlichkeit des Kindes eingeschränkt sein kann. Aussprachestörungen können z.B. durch eine neurologische Grunderkrankung wie beispielsweise der Zerebralparese oder durch Lippen-Kiefer-Gaumen-Spalten, angeborene Hörstörungen oder Behinderungen mit kognitiven Einschränkungen (z. B. Down-Syndrom) verursacht sein. In den meisten Fällen liegt allerdings keine organische Ursache vor. Zu unterscheiden sind Kinder mit einer reinen Laut-Fehlbildung (z. B. Lispeln oder einer lateralen Fehlbildungen des <sch>Lautes, sodass eine Art schlürfendes Geräusch entsteht) von Kindern, die systematische oder sogar unsystematische $\mathrm{Er}$ setzungen und Auslassungen von Lauten zeigen.

Mitte des letzten Jahrhunderts ging man davon aus, dass Aussprachestörungen vor allem durch mangelnde Reifung der Artikulations-Muskeln (z. B. Zunge, Lippen) verursacht werden. Daher stellten mundmotorische Übungen einen zentralen Baustein der Behandlung von Aussprachestörungen nach van Riper (1939/ 1963) dar [2]. Im Gegensatz zur bis heute häufigen therapeutischen Umsetzung des Ansatzes betonte van Riper, dass ein generelles Training aller Artikulationsmuskeln nicht sinnvoll sei, sondern nur Übungen, die direkt einen Laut in seiner Bewegung vorbereiten. So sollen z. B. Übungen zur Stärkung einer spitzen, nach oben zeigenden Zunge die korrekte Bildung des Lautes <s> vorbereiten. Solche spezifischen Übungen können auch heute noch kurzfristig eingesetzt werden, wenn die Bildung eines Lautes, den ein Kind noch nicht produzieren kann, unterstützt werden soll.

Seit Ende der 1960er-Jahre ist allerdings bekannt, dass kindliche Aussprachestörungen in der Regel nicht mit einer muskulären Schwäche oder Fehlfunktion in Zusammenhang stehen, sondern zum Beispiel mit inkorrekter oder mangelnder Analyse oder Speicherung von gehörten Wortformen (Klangbild eines Wortes). Dies bedeutet, dass der therapeutische Schwerpunkt auf der Analyse und Speicherung von Wortformen liegen sollte und nicht auf dem Training rein korrigierender Bewegungsabläufe.

Des Weiteren konnte die Forschung nachweisen, dass Übungen der Zungenund Lippenmuskulatur auch bei Menschen mit einer neurologischen Erkrankung, die zu einer Einschränkung der Bewegungsfähigkeit, verursacht durch mangelnde Steuerung oder eine Schwäche der Artikulationsmuskeln (Dysarth- rie), führen, keinen positiven Einfluss auf die Sprachfähigkeit der Patienten nehmen. Es liegt ein Unterschied zwischen der Sprechmotorik und der Mundmotorik vor, sodass Erfolge in der Sprechmotorik nicht über ein Training der Mundmotorik erreichbar sind. Zahlreiche Untersuchungen konnten zeigen, dass Kinder mit einer nicht organischen Aussprachestörung vom Einsatz genereller mundmotorischer Übungen nicht profitieren. Dies bedeutet, dass die Durchführung von mundmotorischen Übungen einen Therapieerfolg nicht schneller herbeiführt, als wenn auf die Durchführung dieser verzichtet wird. So herrscht mittlerweile ein Konsens unter den Experten für Aussprachestörungen weltweit [3], dass auf ein generelles, regelmäßiges Training der mundmotorischen Fähigkeiten in der Aussprachetherapie verzichtet werden sollte.

\section{FAZIT}

Generelle unspezifische Übungen zur Mundmotorik sind für Aussprachestörungen kein sinnvolles therapeutisches Mittel. An die Bedürfnisse des Kindes spezifisch angepasste Übungen sind zentraler Baustein der myofunktionellen Therapie. Im Bereich Aussprachestörungen können spezifisch ausgewählte Übungen bei Bedarf die Lautanbahnung kurzfristig unterstützen.

\section{Annette Fox-Boyer, Rostock}

\section{Literatur}

[1] Kittel A. Myofunktionelle Therapie. 11. Aufl. Idstein: Schulz-Kirchner; 2014

[2] Van Riper C. Speech Correction: principles and methods. 4th ed. New York: Englewood Cliffs, Prentice Hal; 1939/1963

[3] Fox-Boyer A. Kindliche Aussprachestörungen Erwerb - Differentialdiagnostik - Therapie.

7. Aufl. Idstein: Schulz-Kirchner; 2016

[4] Fox-Boyer A, Groos I, Schauß-Golecki K. Kindliche Aussprachestörungen - ein Ratgeber für Eltern, Erzieher, Therapeuten und Ärzte. 2. Aufl. Idstein: Schulz-Kirchner; 2015 